\title{
Anomalous right subclavian artery and coarctation of the aorta \\ Surgical implications and the use of the right subclavian artery as a flap
}

\author{
J A ODELL, S SPILKIN \\ From the Thoracic Surgical Unit and Cardiology Department, Wentworth Hospital, Durban, South Africa
}

SUMMARY Coarctation of the aorta was repaired surgically in a 4 month old boy using the anomalous right subclavian artery as a flap. The role of the anomalous artery in providing collateral flow to the descending aorta should be determined so that injudicious clamping is avoided.

The anomalous right subclavian artery is the single most common abnormality of aortic arch development and is found at approximately one out of every $200^{1}$ necropsies. Maude Abbott reported an incidence of $1 \%$ (2/200 patients) of an anomalous right subclavian artery associated with a postductal aortic coarctation. ${ }^{2}$ In our experience two out of 106 patients have had this anomaly; the first case has been reported previously $^{3}$ and the second is reported here. To our knowledge, it is the first report of the use of the anomalous right subclavian artery as a flap to repair the coarctation.

\section{Case report}

A 7 week old boy had a heart murmur at a routine postnatal visit. The mother admitted that the child was breathless when feeding. On examination he was tachypnoeic but not cyanosed. The radial pulses were easily felt, but the leg pulses were not palpable. At the left sternal border an ejection systolic murmur and a loud pulmonary component of the second heart sound were heard. There was a short mid-diastolic murmur at the mitral area. The chest radiograph showed an enlarged heart and pulmonary plethora. The child was diagnosed as having a coarctation with a possible ventricular septal defect. Treatment for cardiac failure was started.

At 3 months of age cardiac catheterisation showed a non-restrictive ventricular septal defect and severe

Requests for reprints to Dr J Odell, Thoracic Surgical Unit, Wentworth Hospital, P B Jacobs, Durban, Natal 4026, South Africa. pulmonary hypertension (pulmonary artery pressure $95 / 30 \mathrm{~mm} \mathrm{Hg}$, pulmonary vascular resistance 2.7 units $/ \mathrm{m}^{2}$ ). The aortogram showed the right carotid artery, left carotid artery, and left subclavian artery arising separately and in that order from the aortic arch. The right subclavian artery arose distal to the left subclavian and just proximal to the site of a pronounced coarctation. The distal descending aorta was normal in size. At 4 months of age repair of the aortic coarctation and banding of the pulmonary artery were undertaken. A left thoracotomy was made and the coarctation exposed. Because of the immediate proximity of the anomalous right subclavian artery to the coarctation we decided to use this vessel as a flap. The

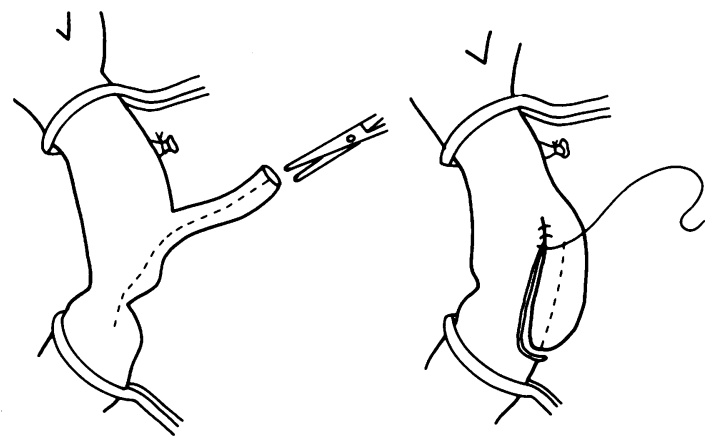

Fig. 1 Diagram showing the surgical technique. The descending thoracic aorta and anomalous right subclavian artery in the region of the coarctation are mobilised. The anomalous right subclavian artery is ligated as far distally as possible and an incision extended along the length of the right subclavian and across the coarctation. The flap is turned down and sutured. 


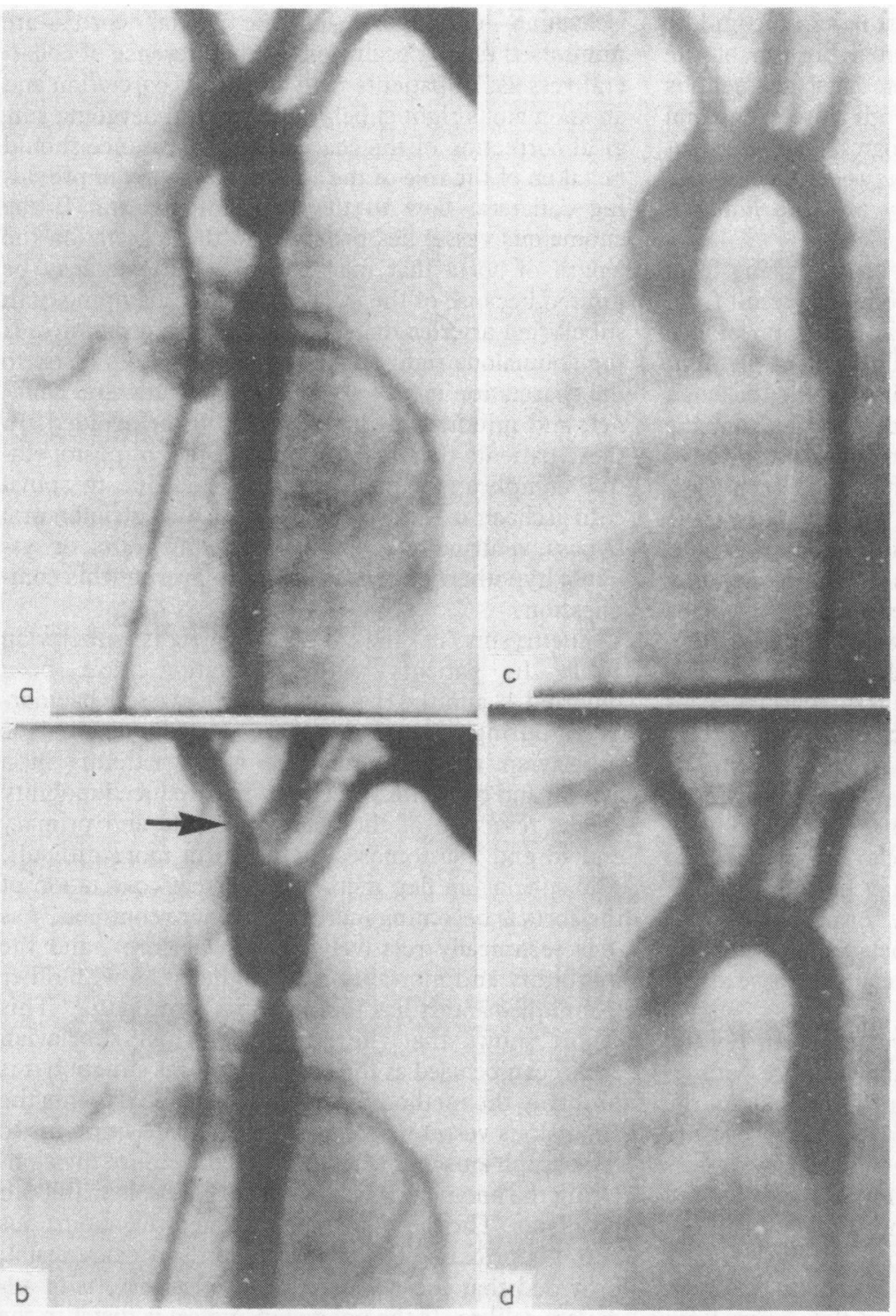

Fig. 2 (a) and (b): preoperative aortograms in a slight left anterior oblique view showing the right carotid, left carotid and left subclavian arteries filling sequentially followed by the anomalous right subclavian (arrow), which arises proximal to an aortic coarctation. (c) and (d): postoperative aortograms in the left anterior oblique view showing no filling of the right subclavian artery and repair of the coarctation.

ligamentum arteriosum was ligated and divided. The aorta was displaced anteriorly and rotated to expose and allow dissection of the anomalous right subclavian artery, which pased medially and posteriorly to the oesophagus. The aorta was cross clamped and the right subclavian artery ligated distally. The subclavian artery was opened along its length and the incision extended across the coarctation into the area of poststenotic dilatation (Fig. 1). The flap of subclavian artery was turned down into the aortic incision and sutured in place with a continuous suture. The pulmonary artery was banded.
The postoperative course was uneventful, and the congestive cardiac failure was easily controlled. The child was recatheterised. There was satisfactory banding of the pulmonary artery, and a small gradient of $15 \mathrm{~mm} \mathrm{Hg}$ across the site of coarctation. Fig. 2 shows the preoperative and postoperative appearances of the thoracic aorta.

\section{Discussion}

The normal right subclavian artery has three embryological components: the most proximal seg- 
ment is derived from the right fourth arch; the middle segment is produced by the cranial portion of the right dorsal aorta; and the most distal segment is developed from the right seventh intersegmental artery. During normal embryological development the right dorsal aorta disappears between the origin of the seventh intersegmental artery and the junction with the left dorsal aorta.

An anomalous right subclavian artery arising from the descending thoracic aorta is thought to result from persistence of the right dorsal aorta and disappearance of the right fourth arch and cranial portion of the right dorsal aorta. Thus the anomalous right subclavian artery, as it originates from the descending thoracic aorta and passes across the mediastinum posterior to the oesophagus, is derived from the right dorsal aorta and right intersegmental artery. Knowledge of the embryological development of this anomaly would suggest that its point of origin is on the descending thoracic aorta distal to the ligamentum arteriosum (a remnant of the left sixth aortic arch) and therefore distal to the site of the usual location for postductal aortic coarctation. When, however, coarctation is associated with an anomalous right subclavian artery the anomalous vessel may be found proximal ${ }^{4-7}$ or distal $^{8-10}$ to the coarctation site. Varying degrees of migration may explain the variance in the point of origin of the anomalous right subclavian artery to the coarctation. Aortic coarctation may be found proximal to both subclavian arteries ${ }^{11-14}$ or proximal to the left common carotid and both subclavian arteries, ${ }^{3}$ or an anomalous right subclavian artery may arise at the site of the coarctation. ${ }^{15}$

In patients with an aortic coarctation and normal development of the arch vessels and in those with an anomalous right subclavian artery proximal to the coarctation, the internal mammary arteries-together with other branches from the subclavian vessels around the scapulae-provide important collateral pathways for blood flow beyond the coarctation. When an anomalous right subclavian artery originates distal to the coarctation this vessel acts as the main collateral channel. Retrograde blood flow ocurs from the right vertebral artery into the subclavian artery and descending thoracic aorta. When both subclavian arteries arise distal to the coarctation interesting features may be seen-namely, the presence of papilloedema ${ }^{12}$ and the absence of a palpable pulse in all four limbs. In this situation the most important collateral circulation is likely to be through the circle of Willis, with retrograde flow along the vertebral and subclavian arteries.

Most techniques for repair of coarctation of the aorta require temporary occlusion of the thoracic aorta. The proximal effect of increased vascular resistance on the heart and the distal effect of ischaemia-particularly of the spinal cord ${ }^{7}$-are minimised during occlusion by the presence of collateral vessels. In patients with an aortic coarctation and an anomalous right subclavian artery undergoing surgical correction of the coarctation, cognisance should be taken of the role of the anomalous artery in providing collateral flow to the descending aorta. If the anomalous vessel lies proximal to the coarctation the length of aorta that may be cross clamped may be limited because of the inadvisability of clamping both subclavian arteries that may be in close proximity. If the anomalous right subclavian artery arises distal to the coarctation it may act as the main collateral channel, and injudicious clamping should be avoided. In these patients there is an increased risk of postoperative complications such as paraplegia due to spinal cord ischaemia, and techniques such as atriofemoral bypass, ventriculofemoral bypass, jump grafts, or systemic hypothermia are advised ${ }^{10}$ to prevent this complication.

Aneurysms of the anomalous right subclavian artery in patients with coarctation have been reported, ${ }^{16}$ and the risk of creating a massive haemorrhage during dissection is increased. The anomalous subclavian artery tethers the aortic isthmus in a medial and posterior direction. This reduced mobility makes resection of the coarctation site and primary end to end anastomoses of the aorta more difficult. The subclavian flap method to correct coarctation of the aorta is becoming increasingly more common, ${ }^{17}$ as it is technically relatively easy to perform, and the morbidity and mortality is no higher than with other techniques and it has the potential of growth. ${ }^{18}$ This report shows that the anomalous right subclavian artery can be used as the flap, and in this situation it is probably the method of choice as in some patients the anomalous vessel may cause obstructive symptoms to the oesophagus and trachea and will require division. Using the anomalous vessel as the flap deals with both problems. The flap may be used when the anomalous vessel is proximal to the coarctation and when distal, provided that the vessel (subclavian artery) is of adequate calibre. If the anomalous vessel is distal to the coarctation due care should be taken to prevent spinal cord ischaemia. ${ }^{19}$ The only theoretical disadvantage of using the anomalous right subclavian artery as a flap is the possible development of a subclavian steal as the vertebral artery is not ligated. This complication is probably uncommon since it is rarely reported after division of the vessel for obstructive symptoms to the trachea or oesophagus.

\section{References}

1 Engelman RM, Madayag M, Spencer FC. Aneurysms of 
aberrant right subclavian artery. NY State f Med 1973; 73: 290-2.

2 Abbott ME. Coarctation of the aorta of the adult type II. A statistical study and historical retrospect of 200 recorded cases, with autopsy, of stenosis or obliteration of the descending arch in subjects above the age of two years. Am Heart F 1928; 3: 381-421.

3 Le Roux BT, Williams MA. An unusual aortic coarctation. Thorax 1968; 23: 640-4.

4 Reid DA, Foster ED, Stubberfield J, Alley RD. Anomalous right subclavian artery arising proximal to a post-ductal thoracic aortic coarctation. Ann Thorac Surg 1981; 32: 85-7.

5 Fawcett J. Coarctation of the aorta as illustrated by cases from the post-mortem records of Guy's Hospital from 1826-1902. Guy's Hospital Reports 1905; 59: 1-19.

6 East T. Coarctation of the aorta. Proc $R$ Soc Med 1932; 25: 796-800.

7 DeBoer, A Grana L, Potts WJ, Lev M. Coarctation of the aorta. Arch Surg 1961; 82: 801-12.

8 Gross RE. Coarctation of the aorta. Surgical treatment of 100 cases. Circulation 1950; 1: 41-55.

9 Griffith GC, Oblath RW, Jones JC. Unusual manifestations of coarctation of the aorta. Circulation 1955; 12: 1080-3.

10 English TAH. Direct left ventriculofemoral bypass during resection of coarctation of the aorta with anomalous subclavian arteries. Thorax 1965; 20: 36-9.
$11 \mathrm{McGregor} \mathrm{M}$, Medalie $\mathrm{M}$. Coarctation of the aorta. $\mathrm{Br}$ Heart f 1952; 14: 531-3.

12 D'Abreu AL, Aldridge AGV, Astley R, Jones MAC. Coarctation of the aorta proximal to both subclavian arteries producing reversible papilloedema. $\mathrm{Br} \mathcal{f}$ Surg 1965; 48: 525-7.

13 Dodrill FD, Benson CD. Coarctation of the aorta with both subclavian arteries arising from the distal segment complicated by post-operative intussusception. Surgery 1962; 51: 809-13.

14 Nathoo AR. Two unusual cases of coarctation of the aorta. Guy's Hospital Reports 1965; 114: 32-7.

15 Partyka LC, French SW, Runer GF. Anomalous innominate and right subclavian arteries associated with coarctation of aorta. Arch Surg 1958; 77: 81-6.

16 Sakurai H, Lukban S, Litwak RS. Coarctation of aorta associated with anomalous right subclavian artery and its aneurysm. NY State f Med 1973; 73: 292-6.

17 Waldhausen JA, Nahrwold DL. Repair of coarctation of the aorta with a subclavian flap. F Thorac Cardiovasc Surg 1966; 51: 532-3.

18 Hamilton DI, Di Eusanio G, Sandrasagra FA, Donnelly RJ. Early and late results of aortoplasty with a left subclavian flap for coarctation of the aorta in infancy. $f$ Thorac Cardiovasc Surg 1978; 75: 699-704.

19 Brewer LA, Fosburg RG, Mulder GA, Verska JJ. Spinal cord complications following surgery for coarctation of the aorta. F Thorac Cardiovasc Surg 1972; 64: 368-81. 